\title{
El Homo sapiens, la fe y el efecto placebo
}

\author{
Homo sapiens, faith and the placebo effect
}

\author{
Dr. C. Jorge A. Bergado \\ Centro Internacional de Restauración Neurológica (CIREN). La Habana, Cuba.
}

\begin{abstract}
RESUMEN
Los llamados efecto placebo y nocebo son efectos reales que resultan de la interacción entre la actividad mental y el estado funcional del organismo. Esta interacción se puede describir hoy en términos precisos a través de la influencia que las estructuras del sistema límbico ejercen sobre el hipotálamo y las regiones del tallo cerebral que controlan las funciones endocrina, motora y vegetativa. El conocimiento de estos mecanismos pone de relieve la importancia de factores sugestivos, como la confianza en el terapeuta o en el tratamiento indicado, en la curación de enfermedades o de sus secuelas. Existen evidencias de que algunas terapias sin una base científica sólida como la acupuntura, la homeopatía o la terapia floral, logran sus resultados a través de estos mecanismos. Incorporar los principios psicobiológicos que origina el efecto placebo a la relación médico paciente, puede resultar una contribución positiva para una medicina más efectiva y humana, pero siempre dentro de los límites que imponen la ética de no mentir y el respeto a la integridad e inteligencia de los pacientes.
\end{abstract}

Palabras clave: placebo, nocebo, sistema límbico, cerebro, mente, cuerpo, relación medico paciente, ética.

\section{ABSTRACT}

The so called placebo and nocebo effects are real, and result from the interaction between the mental activity and the functioning of the body. This interaction is presently described in precise terms as the influence exerted by limbic structures on the hypothalamus and on the brain stem's nuclei that control the endocrine, motor and vegetative functions. Understanding of these mechanisms discloses the important role played by suggestion, like trusting your therapist or trusting the treatment, in the 
cure of diseases and their sequels. There is also evidence that therapies without a strong scientific foundation, like acupuncture, homeopathy or flower therapy, can achieve some results based on these mechanisms. The introduction of the psychobiological principles governing the effect of placebo into the medical practice could contribute to a more effective and human medicine, provided that the ethical limits imposed by the truth and the respect to the patient's integrity and intelligence are observed.

Key words: placebo, nocebo, limbic system, brain, mind, body, medicalpatient relation, ethics.

\section{NTRODUCCI ÓN}

La palabra placebo, viene del latín "placere" que significa complacer -más que placery que, como concepto, ha estado presente en la medicina occidental desde hace tiempo.

En mi caso, la primera vez que oí hablar de un placebo fue hace ya muchos años, mientras pasaba el curso de Sanitario Camillero durante el Servicio Militar. Aunque nunca lo hice o vi hacer, se decía que era el mecanismo infalible para detectar simuladores que se hacían pasar por enfermos o adoloridos para evadir tareas o lograr, eventualmente, su desmovilización. Consistía, en inyectar un preparado inerte (ej. cloruro de sodio o agua destilada) declarando al presunto simulador que se le había administrado un analgésico. Si poco después el individuo se declaraba aliviado, la impostura quedaba descubierta.

Después conocí del placebo como tratamiento de complacencia, aplicado a personas que exigían medicinas y atención sin estar realmente enfermas, y más tarde, del placebo como tratamiento inerte entre los grupos experimentales exigidos en ensayos clínicos rigurosos.

Pero la ciencia progresa y los conceptos evolucionan. En los últimos decenios, a partir del impulso dado por uno de los pioneros en la investigación del placebo, Henry Knowless Beecher (1904-1976), en su obra "The powerful placebo" 1955, citado por Meissner $\mathrm{K},{ }^{1}$ el concepto de placebo ha cambiado, y de inerte cazador de timadores, el llamado efecto placebo adquiere una relevancia creciente en la práctica y en la investigación médica actual.

\section{HOMO SAPIENS}

El hombre es un ser biopsicosocial. Tal es el enunciado que resume los componentes que interactúan en la constitución del hombre en su real dimensión. El Homo sapiens es el componente biológico, base sobre la cual en su desarrollo como parte de la sociedad, ese ente biológico se convierte en un ser humano, que piensa, habla, trabaja, cumple normas, tiene convicciones, aspiraciones, creencias y un largo etcétera. Es claro aquí que la influencia del grupo (educación) actúa sobre las premisas biológicas que le confieren su gran cerebro, sus muy especializados 
sensores, su posición bípeda, su laringe liberada, su gregarismo y otro muy largo etcétera, y es clave para convertir al Homo sapiens en un ser humano.

Sin embargo, hay aspectos de esas relaciones tripartitas que han sido poco tratados. Uno de ellos es la existencia de factores biológicos que limitan, condicionan o dificultan el desarrollo psicológico. Esto, que es cierto en personas con discapacidades físicas o intelectuales, es también un factor presente en personas que no tienen esas graves limitaciones, lo que no significa que no tengan alguna incompetencia. No somos solo una cornucopia

de aptitudes, somos también una gavilla de ineptitudes. La vulnerabilidad inherente a nuestra constitución de seres albuminoideos y perecederos es un elemento que, al ser percibido conscientemente, se constituye en sustrato de temores, supersticiones y otras falsas creencias. ${ }^{2}$ La superstición se nutre no solo de ignorancia, el saber puede ser fuente de sufrimientos e intuyo es esa la alegoría que relata el Génesis cuando condena a los protohumanos Eva y Adán, a sufrir por haber probado el fruto del árbol del conocimiento.

De la misma forma en que lo social determina y condiciona lo psicológico, así también lo psicológico condiciona e influye sobre lo biológico. Abundan los ejemplos en la vida cotidiana de cómo el estado anímico o afectivo de una persona influye y repercute sobre el funcionamiento de sus órganos y sobre su salud. Sin embargo, la influencia de lo psíquico sobre lo corporal solo ha sido reconocida en años recientes, en parte por la persistente costumbre de considerar que la mente es una especie de entelequia inmaterial que flota sobre el cerebro sin ser parte integrante de este. Sin embargo, cuando comprendemos que el alma o espíritu es el producto de la actividad del cerebro, se desarrolla y muere con él, no es difícil concebir que nuestra manera de pensar y los eventos que afectan nuestro ánimo puedan tener una influencia importante y real sobre nuestra fisiología y sobre nuestra salud.

En el conjunto de estructuras nerviosas que forman el llamado sistema límbico están las claves para entender estas reacciones psicosomáticas. Los núcleos del complejo nuclear amigdalino, en lo interno del polo temporal, censan continuamente la información sensorial y establecen la valencia afectiva de cada momento: ¿me gustame disgusta?, ¿es bueno-es malo? El hipocampo conserva temporalmente una huella de memoria que será consolidada y conservada si es reforzada por señales de activación amigdalina. Los mecanismos fisiológicos de esta interacción se conocen en detalle hasta el nivel celular y molecular. ${ }^{3}$ El hipotálamo, en la base del cerebro, modula y modifica la actividad de nuestros sistemas motores: somático y vegetativo, así como la función de todo el sistema endocrino y reproductor a través de sus relaciones con la hipófisis (Fig.).

Se entiende entonces, cómo un estado emocional o motivacional intenso puede modificar la función cardiovascular, los ciclos sexuales o hacer imborrable un contenido de memoria. Se entienden incluso, fenómenos más dramáticos, como los espectaculares cuadros histéricos y los misterios de la hipnosis.

\section{EL EFECTO PLACEBO}

\section{Analgesia y placebos}

La observación de que determinadas circunstancias, por ejemplo el fragor de un combate, pueden reducir la percepción del dolor es muy antigua. En los corros familiares era reiterado, que mientras "la sangre estuviera caliente" la persona apenas sentía el dolor de una herida. El conocimiento popular no vinculaba este efecto con la analgesia por placebo, pero intuía con mucha razón, que algo dentro de nosotros, probablemente en el cerebro, podía obrar ese milagro. El ya mencionado 
Knowless Beecher tuvo ocasión de comprobarlo en los campos de batalla y dedicó sus esfuerzos a intentar comprenderlo.

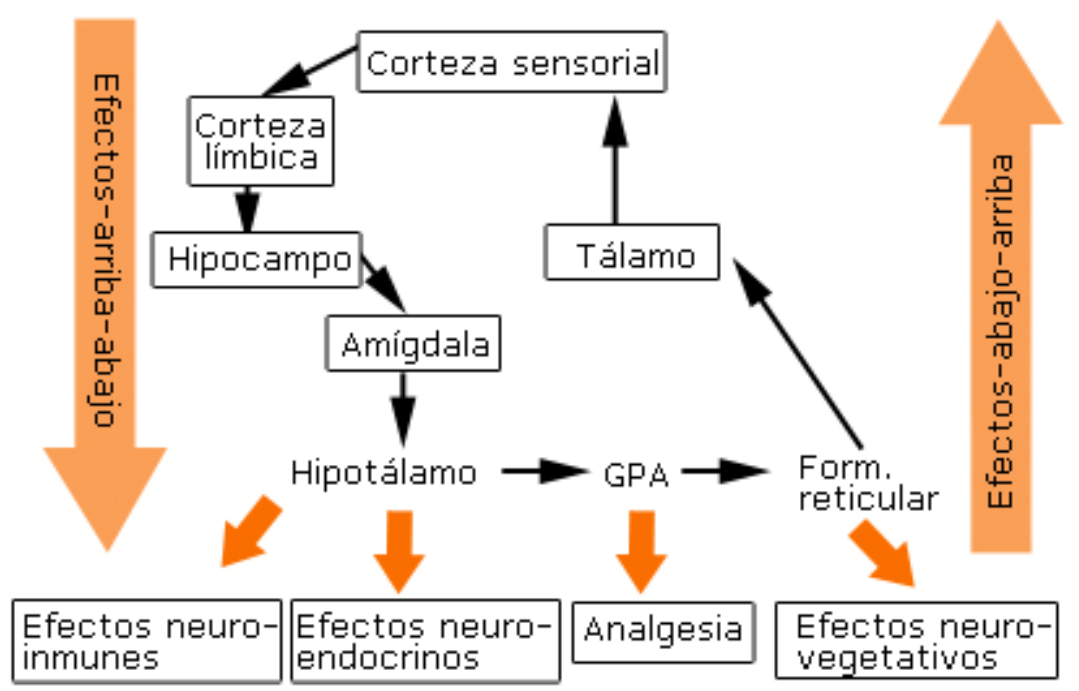

GPA: sustancia gris periacueductal, Form. reticular: formación reticular.

Fig. Se muestran algunas de las estructuras cerebrales relacionadas con el efecto placebo. Las flechas indican las relaciones entre ellas en la producción de reacciones mentales provocadas por influencias corporales (abajo-arriba) o viceversa (arribaabajo).

La publicación en 1978 de que la respuesta a un placebo en pacientes con dolor posquirúrgico se bloquea con naloxona, ${ }^{4}$ es la primera que vincula directamente al placebo con una respuesta cerebral medible. La naloxona es un antagonista de receptores a opiáceos, de modo que el resultado significa que la respuesta placebo implica la activación de sistemas de analgesia interna del tipo endorfinérgico. Este efecto es replicado por Petrovic y otros, ${ }^{5}$ y muestra similitudes entre el uso de placebo y un agonista opiáceo (remifentanil). En 2005, Zubieta y otros, citado por el mismo autor en el $2009,{ }^{6}$ con el uso de técnicas de imaginología molecular, demuestran que la expectativa de alivio del dolor era capaz de activar respuestas dependientes de receptores $\mu$-opiáceos en áreas cerebrales diversas. Estas incluyen: la corteza cingulada rostral y prefrontal dorsal, la ínsula, el núcleo accumbens, la amígdala, el tálamo, el hipotálamo y la sustancia gris periacueductal (Fig.). Algunas, como la última mencionada, han sido identificadas hace tiempo como parte del sistema de analgesia interno. Otras como el accumbens, y la amígdala, sugieren la implicación de componentes motivacionales en la analgesia por placebo.

Dado el carácter eminentemente afectivo del dolor, su relación con el sistema límbico hace muy probable que en la analgesia por placebo intervengan también mecanismos positivos de reforzamiento emocional. ${ }^{7}$

Se han realizado interesantes estudios en la búsqueda de los mecanismos de procedimientos analgésicos no convencionales en la cultura cubana, como la acupuntura. Mientras que varios estudios de imágenes funcionales identifican blancos de activación, comunes a los informados por Zubieta, otros sin embargo, ponen en duda la especificidad de los puntos acupunturales, sugiriendo un mecanismo mediador de tipo placebo. ${ }^{8}$ 


\section{El efecto placebo más allá del dolor}

Descubrir y desentrañar los mecanismos fisiológicos de la analgesia por placebo constituyó un acicate para que se explorara la participación de otros mecanismos en relación con los placebos. Obviamente, el mal llamado sistema nervioso autónomo, ha sido uno de los objetos de tales estudios. En un artículo publicado en un número especial de las Transacciones Filosóficas de la Real Sociedad dedicado al placebo, Meissner y otros, ${ }^{9}$ refieren los resultados de varias investigaciones propias y de otros, que demuestran que la expectativa de un tratamiento puede modificar funciones circulatorias (la presión sistólica, por ejemplo), digestivas (cómo la motilidad gástrica), respiratorias (el grado de constricción de las vías aéreas) y otras dependientes del sistema neurovegetativo. Estos resultados confirman varias cosas que sabíamos desde hace mucho tiempo: que no se quiere con el corazón aunque lo parezca, que nos podemos orinar o defecar de puro miedo, y confirman además que el sistema nervioso autónomo no es en absoluto independiente, sino que forma parte de un sistema de control de las funciones vegetativas que cuenta con receptores, vías aferentes, centros nerviosos, vías eferentes simpática y parasimpática) y efectores. Y aunque resulta realmente difícil modificar voluntariamente las funciones que controla (se cuenta que el entrenamiento yoga ayuda a hacer esto), es también muy cierto que el estado emocional y el estrés pueden provocar cambios agudos o crónicos en estas funciones y provocar una enfermedad orgánica (el llamado efecto nocebo) o, por el contrario, contribuir a su curación. Un ejemplo bien estudiado parecer ser el uso de acupuntura para reducir la presión arterial. Como en el caso de la analgesia, se comprueba que introducir agujas puede reducir significativamente los valores de presión arterial, pero sin diferencia con el grupo en que se usaron puntos no acupunturales. ${ }^{10}$

Los efectos del placebo se basan en dos mecanismos diferentes pero complementarios. Uno es la expectativa de la persona, buena o mala, ante un tratamiento o maniobra. El otro concierne a efectos de condicionamiento pavloviano clásico. ${ }^{11}$ La expectativa de mejoría o curación, genera un estado particular de activación en estructuras límbicas que refuerzan no solo los efectos de un placebo, ${ }^{12}$ sino otros procesos mentales como la memoria, o neurológicos como la efectividad de la neurorrehabilitación. ${ }^{13}$ Una diferencia fundamental entre ambos mecanismos es que en la primera, el sujeto es consciente de la expectativa y puede transmitirla verbalmente a otros, mientras que la segunda solo puede ser adquirida por exposición directa del sujeto al condicionamiento. ${ }^{12}$

Los mecanismos de condicionamiento pueden darse en reacciones autonómicas como las descritas arriba y también en funciones del sistema inmune. En un estudio clásico en cobayos, ${ }^{11}$ los investigadores aplicaron a cobayos repetidas veces una sustancia natural que eleva el conteo leucocitario en sangre. Cada aplicación de la sustancia (el estímulo incondicionado en este caso), se asoció a la aplicación de calor en una zona de la piel. Luego de varias repeticiones, la aplicación de calor, por si sola, elevó el conteo leucocitario, convirtiéndose así en el estímulo condicionado de una respuesta inmune. Este mecanismo de condicionamiento funciona igual que cualquier otro reflejo (recordar los perros de Pavlov) y sus componentes centrales son atribuidos a la corteza insular. Lo menos conocido es el medio por el cual el cerebro censa el cambio en la actividad inmune y se condiciona para producirla. La primera podría estar mediada por la acción de las propias citocinas inductoras de la respuesta inmune y permeables a la barrera hematoencefálica. La señal eferente parece mediada por señales noradrenérgicas simpáticas hacia órganos esenciales del sistema inmune como el bazo.

Las implicaciones clínicas de esta relación entre el sistema nervioso y el sistema inmune y su condicionamiento dependiente de la experiencia son diversas. Por una parte, pueden intervenir en el desarrollo de enfermedades tan comunes como las 
alergias (incluida el asma bronquial) o tan devastadoras como el lupus eritematoso; de la otra ya se especula sobre la posibilidad de lograr una depresión condicionada de la inmunidad en pacientes candidatos a recibir trasplantes de órganos.

\section{Reconceptualizando el placebo}

El progreso científico ha permitido no solo demostrar que las respuestas a un placebo existen, son reales y no siempre simuladas, sino también identificar factores que la modifican como las expectativas, la motivación, la espiritualidad y el condicionamiento pavloviano. Y más aun, nos permite identificar bases neurobiológicas para esta respuesta.

Este conocimiento impacta a la práctica y la investigación médicas de muchas maneras. El ensayo clínico a doble ciegas, controlado contra placebo, sigue siendo el máximo nivel de evidencia sobre la efectividad de una droga o intervención. Sin embargo, resulta necesario tener en cuenta que el efecto placebo puede modificar considerablemente los resultados de ese grupo experimental. Existe evidencia innegable de que la probabilidad percibida por el sujeto experimental de estar en el grupo "activo" incrementa el efecto del placebo. Algo que también depende mucho del tipo de instrucción que se ofrece a los participantes durante la entrevista de enrolamiento en el estudio. El uso de placebos que mimetizan algunos efectos colaterales conocidos de la droga bajo estudio, también incrementa el efecto del placebo. Los placebos físicos, como la pseudoacupuntura son más efectivos que los placebos farmacológicos. ${ }^{1,14-18}$ Incluir medidas para controlar estos efectos es un imperativo creciente de la investigación médica actual.

Por otra parte, la demostración de que el efecto placebo es real, puede incrementar la tentación de los terapistas profesionales (médicos, psicólogos, rehabilitadores y otros) de emplear placebos, sin considerar el dilema ético que tal proceder supone. Emplear deliberadamente un placebo casi nunca será una mentira piadosa. En su lugar Jonas ${ }^{19}$ propone maximizar el efecto terapéutico mediante acciones asociadas al tratamiento farmacológico o quirúrgico. Entre ellas, algunas son ajenas a nuestra sociedad, como la de recetar productos de marcas muy reconocidas y publicitadas. Otras son de una eticidad cuestionable, como la de asociar condicionantes benignos (como un aroma o un ritual) al tratamiento. Pero muchas son acertadas y lamentablemente obviadas muchas veces en la vorágine de una demanda desbordante:

- Utilice siempre el mejor tratamiento posible y crea en el tratamiento que aplica.

- Aplique el tratamiento en un ambiente cálido y afectuoso.

- Aplique el tratamiento inspirando confianza y credibilidad.

- Informe al paciente de cuales son los resultados esperados y observables.

- Incorpore al tratamiento métodos de relajación y reducción de la ansiedad.

- Escuche y brinde empatía y comprensión.

- Toque al paciente.

La simplicidad de las recomendaciones las convierte en algo casi trivial: itoque al paciente! Lamentablemente tengo experiencias, como paciente o acompañante, de la violación flagrante de estas simplezas en nuestros centros de atención médica. Es 
curioso que luego de tantas vueltas y revueltas, alguien tenga que recomendar cosas que todos los buenos médicos saben que forma parte de eso que se llama "el arte de curar". La espiral del progreso tiene mucho de ese redescubrir el agua tibia. No fue Freud quien descubrió el valor terapéutico de la confesión, fue la Iglesia Católica hace ya un par de milenios.

\section{Del placebo al New Age}

El efecto placebo es un ejemplo de reacción psicobiológica, o psicosomática. Un ejemplo de como nuestras convicciones, motivaciones y expectativas pueden influir sobre nuestro cuerpo y sus funciones y una manera de entender con racionalidad el aparente efecto de terapias tan descabelladas como la radiestesia* o la homeopatía. ${ }^{20,21}$

Aunque no es el único ejemplo disponible, tal vez por el largo camino andado para descubrir sus intríngulis, por las complejidades de su basamento neural y por el cambio conceptual que tales descubrimientos han significado, algunos piensan que es preciso redimensionar el placebo. ${ }^{19,22,23}$

Como en todos los andares de la vida, seamos cuidadosos; porque este camino, como todos, tiene encrucijadas, baches y algunas trampas. En primer lugar no creo que se requiere cambiar la dimensión terapéutica del placebo, porque no es el agente placebo quien tiene algún efecto demostrado, sino la forma en que la persona que lo recibe asume, valora y tiene fe en que ese tratamiento contribuirá a mejorar su condición. ${ }^{24-26}$ Es ese algo a veces llamado relación mente cuerpo, un concepto lamentablemente muy mal tratado por casi toda la literatura no científica y peor tratado por una pléyade de iluminados quienes al enterarse de su existencia, piensan deslumbrados que han descubierto la piedra filosofal de la salud humana. Los ejemplos abundan: desde la terapia floral de Bach hasta otras que ya nos intentan penetrar como la llamada "Descodificación biológica" ${ }^{\dagger}$, inspirada en la Nueva Medicina Germana ${ }^{\ddagger}$.

Esta última fue inventada por el Dr. H. G. Hamer, un médico alemán, quien tras sufrir la pérdida accidental de un hijo y padecer después un cáncer de testículo, desarrolló una alambicada teoría que se basa en leyes o principios de la interacción mente cuerpo, y en los que mezcla conocimientos de Fisiología con especulaciones delirantes y que son la verdad, toda la verdad, la única verdad de la salud y de la enfermedad. La aplicación de estos conceptos ha provocado la muerte de varias personas que han abandonado tratamientos convencionales de cáncer. Hoy Hamer es un prófugo de la justicia, y desde una página web en Internet defiende con fervor paranoico la autenticidad y validez de su Nueva Medicina Germana. Su megalomanía le ha llevado a registrar como marca la Nueva Medicina Germana y a fustigar como plagiarios a otros iluminados que siguen o comparten sus desvaríos, incluida la Descodificación biológica, esto, sin embargo, no es óbice para que los creadores de la Biodescodificación la reconozcan entre las principales fuentes de su doctrina. La Universidad Médica de La Habana, auspició hace poco un curso de posgrado sobre esa Nueva Doctrina de la Nueva Era, lo que es una muestra de que no somos inmunes a estos desvaríos pseudocientíficos. Para defender el bien ganado prestigio de nuestra medicina y, sobre todo, la salud de nuestra población, resulta imperativo establecer una clara y fuerte política de evaluación y aprobación de cualquier nueva terapia, incluidas aquellas que se agrupan bajo la categoría de Medicina Natural y Tradicional. ${ }^{27}$

El efecto placebo es el resultado de la influencia de la psiquis sobre funciones corporales y puede lograr analgesia, alteraciones vegetativas o inmunes a partir de 
sustancias o tratamientos inertes o carentes por si mismos de tales efectos. Esa influencia no es despreciable y puede ser utilizada benéfica y éticamente por cualquier terapeuta mediante la creación de un clima de confianza (en todos los buenos sentidos del término) con las personas que trata. También es un elemento que resulta necesario considerar en el diseño de ensayos clínicos controlados contra placebo, de modo que sus componentes puedan ser debidamente aleatorizados y no sesguen los resultados.

Actualizar nuestros conceptos sobre el efecto placebo sobre firmes bases científicas nos servirá también para eludir las trampas pseudocientíficas que se nos tiendan desde la hipertrofia de la relación mente cuerpo. Ni esta relación, ni el efecto placebo son panaceas, aunque si pueden ser excelentes auxiliares en el tratamiento de muchas dolencias.

\section{REFERENCI AS BI BLI OGRÁFICAS}

1. Meissner K, Kohls N, Colloca L. Introduction to placebo in medicine: mechanisms and clinical implications. Philos Trans R Soc Lond B Biol Sci. 2011;366:1783-9.

2. Wolpert L. Biology of belief. Berichte und Abhandlungen Berlin-Brandenburgische Akademie der Wissenschaften. 2006;10:93-120.

3. Bergado JA, Frey S, López J, Almaguer-Melian W, Frey JU. Cholinergic aferents to the locus coeruleus and noradrenergic afferents to the medial septum mediate LTPreinforcement in the dentate gyrus by stimulation of the amygdala. Neurobiol Learn Mem. 2007;88:331-41.

4. Levine J, Gordon N, Fields $\mathrm{H}$. The mechanism of placebo analgesia. Lancet. 1978;2:654-57.

5. Petrovic $P$, Dietrich $T$, Fransson $P$, Andersson J, Carlsson K, Ingvar M. Placebo in emotional processing- induced expectations of anxiety relief activate a generalized modulatory network. Neuron. 2005;46:957-69.

6. Zubieta JK, Stohler CS. Neurobiological mechanisms of placebo responses. Ann N Y Acad Sci. 2009;1156:198-210.

7. Flaten MA, Aslaksen PM, Lyby PS, Bjorkedal E. The relation of emotions to placebo responses. Philos Trans R Soc Lond B Biol Sci. 2011;366:1818-27.

8. Campbell A. Point specificity of acupuncture in the light of recent clinical findings and imaging studies. Acupunct Med. 2006;24:118-22.

9. Meissner $\mathrm{K}$. The placebo effect and the autonomic nervous system: evidence for an intimate relationship. Philos Trans R Soc Lond B Biol Sci. 2011;366:1808-17.

10. Macklin EA, Wayne PM, Kakish LA, Valaskatgis P, Thompson J, Pian-Smith MCM, et al. Stop hypertension with acupuncture research program (SHARP): results of a randomized, controlled clinical trial. Hypertension. 2006;48:838-45.

11. Vits S, Cesko E, Enck P, Hillen U, Schadendorf D, Schedlowski M. Behavioural conditioning as the mediator of placebo responses in the immune system. Philos Trans R Soc Lond B Biol Sci. 2011;366:1799-807. 
12. Hyland ME. Motivation and placebos: do different mechanisms occur in different contexts? Philos Trans R Soc Lond B Biol Sc.i 2011;366:1828-37.

13. Bergado Rosado JA, Almaguer Melian W. Mecanismos celulares de la neuroplasticidad. Rev Neurol. 2000;31:1074-95.

14. Colagiuri B. Participant expectancies in double-blind randomized placebocontrolled trials: potential limitations to trial validity. Clin Trials. 2010;7:246-55.

15. Enck $\mathrm{P}$, Klosterhalfen $\mathrm{S}$, Weimer $\mathrm{K}$, Horing B, Zipfel S. The placebo response in clinical trials: more questions than answers. Philos Trans R Soc Lond B Biol Sci. 2011;366:1889-95.

16. Hrobjartsson A, Gotzsche PC. Placebo interventions for all clinical conditions. Cochrane Database Syst Rev. 2010;1:CD003974.

17. Koog YH, We SR, Min BI. Three-armed trials including placebo and no-treatment groups may be subject to publication bias: systematic review. PLoS One. 2011;6:e20679.

18. Walach H. Placebo controls: historical, methodological and general aspects. Philos Trans R Soc Lond B Biol Sci. 2011;366:1870-8.

19. Jonas WB. Reframing placebo in research and practice. Philos Trans R Soc Lond B Biol Sci. 2011;366:1896-904.

20. Lewith G, Barlow F, Eyles C, Flower A, Hall S, Hopwood V, et al. The context and meaning of placebos for complementary medicine. Forsch Komplementmed. 2009;16:404-12.

21. Perry R, Terry R, Ernst E. A systematic review of homoeopathy for the treatment of fibromyalgia. Clin Rheumatol. 2010;29:457-64.

22. Díaz-Novas Gómez J. El placebo: luces y sombras. Rev Habanera Ciencias Med [Internet]. 2008 [citado 5 Ago 2012];7(4). Disponible en:

http://scielo.sld.cu/scielo.php?script=sci arttext\&pid=S1729$\underline{519 \times 2008000400011 \& \operatorname{lng}=\text { es }}$

23. Miller FG, Kaptchuk TJ. The power of context: reconceptualizing the placebo effect. J R Soc Med. 2008;101:222-5.

24. Beauregard M. Mind does really matter: Evidence from neuroimaging studies of emotional self-regulation, psychotherapy, and placebo effect. Prog Neurobiol. 2007;81:218-36.

25. Benedetti F, Carlino E, Pollo A. How placebos change the patient's brain. Neuropsychopharmacology. 2011;36:339-54.

26. Colloca L, Miller FG. Role of expectations in health. Curr Opin Psychiatry. 2011;24:149-55.

27. Rojas F, Silva LC, Sansó F, Alonso P. El debate sobre la medicina natural y tradicional y sus implicaciones para la salud pública. Rev Cubana Salud Pública. De próxima aparición 2013.

http://scielo.sld.cu 
* Disponible en: http://es.wikipedia.org/wiki/Radiestesia

† Disponible en: http://www.descodificaciónbiológica.es/

F Disponible en: http://www.newmedicine.ca/spanish-intro.php

J orge A. Bergado. Centro Internacional de Restauración Neurológica (CIREN). Ave. 25 No. 15805 Cubanacán, Playa 11300. La Habana, Cuba.

Correo electrónico: jorge.bergado@infomed.sld.cu 